\title{
LES RITES DE PASSAGE LORS DE LA SEPARATION PARENTS-ENFANT
}

\author{
par \\ Gaétane Lefebre \\ et \\ Monique Morval \\ Université de Montréal
}

\begin{abstract}
RESUME
Cette étude explonatoire de type clinique vise à explorer les modes, raisons et conditions entourant les départs de la famille d'origine, au moyen de 10 études de cas portant sur les parents. Les résultats indiquent que le mariage semble le rite de passage par excellence pour délimiter le réelle séparation parents-enfant, du moins du point de vue des parents. La cohabitation favorise un détachement progressif mais reste un état de transition. Enfin, les rites choisis sont l'indice des fluctuations que subit le système familial.
\end{abstract}

L'exploration des modes choisis pour quitter (se séparer d'avec) la famille d'origine mène à une réflexion sur le mariage et la cohabitation. Nous remarquons l'intérêt grandissant pour cette dernière pratique, celle-ci étant pourtant quasi-inexistante dans les générations antérieures. La présente étude, de type clinique, s'inscrit dans une recherche plus vaste portant sur l'adaptation du couple lors du départ des enfants (Lefebvre 1981) et permet de voir le rôle important que jouent les rites de passage dans le processus de séparation parents-enfant.

Stierlin (1974) considère en effet qu'il y a un travail de libération à effectuer, impliquant la combinaison du présent et du passé interactionnel et la différenciation de soi. Le départ des enfants apparait comme une période privilégiée concernant la résolution de conflits non liquidés, car il réactive l'expérience passée et permet d'en travailler les aspects problématiques (Boszormenyi-Nagy, 1965). Si le parent n'a pas réusi adéquatement la séparation d'avec ses propres parents, il y a de grandes chances pour que les difficultés éprouvées soient reproduites lors de la séparation d'avec ses enfants. Lowenthal et al. (1975) et Peskin (1977) font cependant remarquer que la reviviscence du passé existentiel des parents est fortement influencée par les besoins et circonstances actuelles. D'oû l'importance d'étudier comment s'effectue le passage.

C'est l'anthropologue Van Gennep (1909) qui, le premier, observe les rites de passage dans les tribus primitives. De trois types, ils marquent les phases de transition du cycle de vie:

- les rites de séparation on lieu avant ou pendant le départ: le sujet s'engage personnellement et signifie ainsi la séparation d'avec ses relations et rôles antérieurs:

- les rites de transition impliquent que l'individu se trouve entre deux situations, sans statut propre ou défini: cette étape symbolise la désintégration et entraine l'isolement;

- les rites d'incorporation expriment l'intégration dans une nouvelle identité so- 
ciale: ces rites démontrent une union avec une personne, un nouveau groupe, une nouvelle situation.

Pour sa part, Yeats (1979) répartit les différents rites tribaux en deux catégories selon leurs propriétés fonctionnelles. Les rites homéostatiques correspondent à la perspective traditionnelle et visent à la régulation et au maintien de l'état de groupe (Vizedom, 1976). Par ailleurs, les rites homéorhésiques sont des rites spécifiques liés au développement. Les rites de passage correspondent à cette deuxième catégorie. Ils visent principalement la régulation des changements de rôle et de statut (Hanchett et Casale. 1976; Linton, 1942). Ils fonctionnent non seulement pour restaurer l'homéostasie du système mais pour établir un nouvel équilibre qui incorpore la modification.

Bien qu'il n' $y$ ait pas de consensus quant à sa définition, le rite est généralement décrit comme un acte prescrit, rigide, répétitif et stéréotypé. Pour leur part, les coutumes sont définies comme des habitudes sociales. Elles concernent des comportements répétitifs qui ne sont toutefois pas hautement stylisés. Une polémique s'élève actuellement entre les anthropologues sur la question de savoir si la dimension religieuse doit nécessairement être présente. Yeats (1979) souligne qu'il est crucial pour l'application du rite à l'étude de la famille moderne, vu sa nature largement séculière, de baser la définition du rite sur ses fonctions et les schèmes de comportements plutôt que sur la dimension mystique et religieuse.

Selon cet auteur, les rites familiaux peuvent se répartir en deux catégories: les rites d'union et les rites du cycle de vie. Les rites d'union correspondent à la définition anthropologique du rituel comme étant répétitif. rigide et prescrit. Ils visent le maintien de la structure, c'est-à-dire l'aspect homéostatique du fonctionnement familial. Les activités familiales spéciales (souper dominical, voyages en famille, rassemblements pour les fêtes) ainsi que les loisirs pratiqués par les membres d'une même famille s'avèrent de cet ordre.
Pour leur part, les rites du cycle de vie ont une fonction analogue aux rites de passage dans la culture tribale; ils peuvent être dé. crits comme des actes que ponctuent et affirment les changements liés au développement dans la famille. Ils redéfinissent les statuts des membres et modifient les normes et règles selon de développement de ces derniers. Lorsqu'une transformation liée au développement est affirmée par un rite du cy. cle de vie, la réalité et la signification de la modification sont permises au niveau du conscient de tous les membres, évitant ainsi l'isolement et les réponses négatives. Des conceptions similaires sont avancées par Levy-Strauss (1963), Kitahara (1974) et Friedman (1980). Ces rites fonctionnent pour valider le changement dans la famille et pour en faire une réalité partagée. Ils focalisent l'expression des affects soulevés par le processus de développement et contribuent à l'enrichissement de la vie familiale. Ainsi, les rites du cycle de vie peuvent être vus comme homéorhésiques, car ils facilitent un nouvel équilibre à un niveau supérieur de différenciation dans le systềme familial. Les célébrations de fête et d'anniversaire, les mariages et l'attention particulière dévolue à l'obtention d'un diplôme ou d'un premier emploi peuvent étre considérés comme des rites de ce type.

Deux critères distinguent les rites familiaux des éléments qui composent la routine familiale: la spécificité temporelle et le caractère spécial de l'événement (Yeats, 1979). Notons néanmoins que les rites dans la famille moderne en comparaison avec les rites tribaux sont plutôt des mini-rituels non religieux. Ce sont des événements semistructurés, analogues aux rites tribaux quant à la forme et à la fonction mais non comparables en intensité, religiosité, rigidité et durée (Bloom, 1977; Yeats, 1979).

Devant l'intérét de la question et le peu de documentation disponible concernant les rites du cycle de vie familiale, la présente étude vise à explorer les modes, raisons et conditions entourant les départs de la famille d'origine, de façon à dégager les rites 
employés ainsi que leurs modalités.

\section{Description de l'étude}

Il s'agit d'une étude clinique portant sur les perceptions des parents quant au départ de leurs enfants. Dix couples canadiens français de niveau socio-économique moyen ou supérieur ont été interviewés. Le dernier enfant a quitté le domicile familial depuis au plus cing ans et le mari occupe un emploi à temps plein. Six femmes sont actuellement sur le marché du travail dont une à temps partiel. Parmi les quatre autres, deux ont ressenti le besoin de retourner temporairement au travail lors du départ des enfants. Les partenaires des couples ont vécu ensemble les différentes phases du cycle de vie familiale.

Une entrevue semi-structurée a été réalisée avec chacun des conjoints. En effet, devant le peu de renseignements disponibles concernant la période postparentale et plus précisément les rites de passage, l'emploi d'une méthodologie de type exploratoire s'est avéré tout indiqué. Différents aspects ont été abordés: 1 . les perceptions et sentiments face aux départs successifs des enfants de la maison; 2. les modifications et difficultés entourant ces départs; 3 . les raisons, modes et conditions sous lesquels se sont réalisées les séparations. Les mễmes dimensions ont été investiguées pour le départ des parents de leur propre famille d'origine. Des études de cas ont ainsi été réalisées pour chaque couple.

\section{Etudes de cas}

Il semble que les rites de passage obéissent à un schème familial. D'une façon génErale, les enfants adoptent en effet le même type de rites que leurs parents. Quand ce n'est pas le cas (lorsqu'il y a difficulté de séparation), les enfants d'une même famille ont tendance à choisir le même rite. Par ailleurs, les rites de passage retenus sont 1 'indice des fluctuations que subit le système familial. Nous allons examiner chacun de ces points, en donnant des exemples.

\section{Schème familial}

Dans six familles, nous retrouvons chez les enfants le même schème de passage que chez leurs parents. Cependant, chaque famille agit différemment et les membres d'une même famille procèdent parfois de façon distincte. Même si, traditionellement, les rites de passage sont perçus comme dépendants de la culture (Hanchett et Casale. 1976), c'est la famille qui choisit les rites à utiliser en accord avec ses caractéristiques idiosyncratiques, déterminant ainsi la qualité émotionnelle des passages. La séparation n'est considérée comme définitive par les parents que lorsque l'enfant passe à travers un rite bien structure tel que le mariage. A un niveau moindre cependant, l'obtention d'un emploi à temps plein peut aussi signifier l'accès au réel statut d'adulte.

\section{Cas 1: La famille A}

La famille A se compose de trois enfants: Francine ( 32 ans). François ( 30 ans) et Louise (28 ans). Une transmission intergénérationnelle du processus de séparation s'observe tant entre les départs de la mère et de François qu'entre ceux du père et de Francine. Ainsi, en ce qui a trait à la première paire, les deux séparations d'avec la famille d'origine sont vécues difficilement et apparaissent comme particulièrement brusques. Dans les deux situations, elles se produisent avant l'ảge de 20 ans. François et Madame entretiennent tous deux des relations chaotiques avec leurs parents, attitude qui semble persister, à un degré moindre cependant. Tous deux quittent pour vivre seuls en appartement. Madame et François demeurrent néanmoins dans un entourage de connaissances. Les relations avec les parents semblent s'améliorer à la suite de leur mariage respectif.

Il est aussi possible de dégager des ressemblances entre le départ de Francine et de Monsieur. En effet, ils ont quitté leur famille d'origine afin de travailler à l'extérieur. Dans les deux cas, le départ est perçu comme relativement simple, prévu à l'a- 
vance et soutenu par les parents qui en endossaient les raisons. Les relations avec les parents, antérieures et postérieures au départ, sont décrites comme excellentes. Tous deux laissent leur famille pour se retrouver entourés d'amis.

Pour sa part, Louise quitte pour se marier. Nous remarquons done que les cinq membres de la famille adoptent ultimement le mariage comme rite de passage. Cette situation ne saurait être indépendante de l'attitude présentée par les parents. Bien que François et Francine partent de la maison avant le mariage, la séparation réelle n'est rapportée par les parents que lorsqu'ils se marient. Le mariage sert donc comme seul point de référence pour délimiter une séparation claire et définitive.

\section{Cas 2: La famille B}

La famille B comprend deux enfants: Paul (23 ans) et France ( 22 ans). A 22 ans, Madame quitte sa famille pour travailler à environ 425 milles de chez elle. Elle demeure dans cette ville et s'y marie à 27 ans. A 12 ans, Monsieur est placé dans un pensionnat situé à plus de 100 milles de chez lui. Lorsqu'il a is ans, il abandonne le Collège pour travailler à une vingtaine de milles de sa famille d'origine sans y habiter toutefois. Il se marie a 27 ans. France (17 ans) part du domicile familial pour parfaire ses études à plus de 500 milles de chez elle. Elle travaille maintenant dans cette ville. Paul (20 ans) se sépare d'avec sa famille d'origine lorsque ses parents sont transférés à plus de 400 milles de là, afin de ne pas quitter son emploi. Il se marie environ un an plus tard.

Il ressort que, pour ces parents, le mariage est nettement associé avec une séparation claire et définitive. $\mathrm{A}$ un niveau moindre cependant, l'obtention d'un poste permanent peut aussi octroyer le statut d'adulte, puisque tous les membres de cette famille ont quitté le domicile familial pour travailler.

\section{Les difficultés de séparation d'avec la famille d'origine}

Dans quatre familles, les rites de passage utilisés par les enfants se distinguent consi. dérablement de ceux employés par les parents. Lors du départ, les enfants de la méme famille, à l'exception d'un représentant, optent toutefois pour le même rite soit la co. habitation. Ces familles présentent certaines similitudes: 1) les parents insistent sur le fait que leurs enfants possèdent toujours une clé du domicile familial, symbole d'un év. entuel retour; 2) dans chacune des familles, un des enfants adopte un rite distinct de sa fratrie; 3 ) dans tous les cas, un des enfants présente des difficultés à quitter sa famille, celles-ci s'exprimant principalement par des départs-retours successifs ou par la remise du départ à plus tard. Les parents mentionnent enfin la nécessité de s'adapter aux moeurs et usage nouveaux.

A titre d'hypothèse, et selon les propositions avancées par Friedman (1980), nous pouvons supposer que ces différents éléments indiquent des difficultés au niveau de la séparation. Cet auteur conçoit en effet la cohabitation comme l'expression symptomatique de problèmes non résolus avec la famille d'origine. De plus, il rapporte que la culture et les coutumes peuvent servir d'indicateurs quant aux relations entre les divers membres de la famille. En ce sens, l'enfant qui differe et respecte les coutumes apparaitrait comme le garant des valeurs de ses parents. Enfin, Friedman souligne que le fait de référer à une explication culturelle (par exemple, la nécessité de s'adapter aux moeurs modernes) semble constituer un déni du processus familial.

\section{Cas 3: La famille C}

La famille C comporte trois enfants? Monique ( 28 ans), Anne ( 23 ans) et Danielle (18 ans). Madame et Monsieur ont quitté la maison parentale pour se marier. De son côté, à 23 ans, Monique part du domicile familial afin de cohabiter avec son ami. Anne, à 16 ans, quitte ses parents pour s' installer 
avec des amis près du Collège où elle étudie. Environ dix-huit mois plus tard, elle décide de demeurer seule avec son ami. Daniel, pour sa part, se sépare d'avec sa famille afin de prendre des cours spécialisés. Depuis son départ, il y a un an, il est revenu pour des séjours plus ou moins longs variant de quelques jours à un mois. Son prochain retour est prévu dans trois mois où il se réinstallera avec ses parents afin d'entreprendre des études collégiales.

Tant Madame que Monsieur rapportent que "dans leur temps", quitter la maison parentale pour des motifs autres que le mariage était inadmissible. Madame affirme bien accepter la cohabitation. Toutefois. pour exprimer ses émotions et sentiments face aux départs de ses filles, elle prend le mariage comme point de référence. Monsieur présente la situation autrement; il considère que l'union libre se révèle à la base contre ses croyances. Néanmoins, il admet aujourd'hui la situation, insistant sur la nécessité de flexibilité et d'acceptation des moeurs actuelles.

L'étude du départ de Daniel se révèle particulièrement intéressante. Son départ correspond en effet aux aspirations, aux désirs et pratiquement aux indications plus ou moins conscientes de son père. Daniel et Anne se séparent tôt d'avec leurs parents. Monsieur valorise d'ailleurs la séparation en bas âge qu'il perçoit comme représentant initiative et temperament; cette situation facilite selon lui le développement et la maturité. Bien qu'ils favorisent l'expérimentation de la séparation, les parents paraissent hésiter devant l'aspect définitif du départ. Chaque enfant possède toujours une clé du domicile familial; Madame et Monsieur semblent espérer un retour éventuel, ce qui se conerétise dans le cas de Daniel. Nous remarquons que les enfants manifestent leur séparation d'avec leur famille d'origine par des rites différents de ceux adoptés par leurs parents. Monique, ayant maintenant mis fin à la cohabitation, demeure seule en appartement. Pendant I'été, elle rejoint fréquemment ses parents à leur maison de cam- pagne, passant pratiquement la saison avec eux. Pour sa part, la cohabitation d'Anne s'est consolidée par la venue d'un enfant. Daniel demeure toujours en période transitionnelle.

\section{Les rites indiquent des fluctuations du système familial}

Selon Friedman (1980), les rites de passage sont bien souvent non arbitraires contrairement à ce qu'ils pourraient paraitre. Ils sont toujours partic intégrante d'autres évenéments familiaux indiquant des fluctuations des processus émotionnels. Les rites de passage se produisent au moment où ils représentent le point culminant des affects contenus dans le réseau. Ces mouvements significatifs commencent longtemps avant l'événement-clé et se terminent longtemps après.

Différents auteurs s'entendent pour reconnaitre que l'expérience vécue par le parent lors de sa séparation d'avec sa famille d'origine constitue l'expérience première sur laquelle repose la séparation parentsadolescent (Bloom, 1977; Stierlin, 1974). Ce stade réactive l'expérience passée et permet éventuellement la résolution de conflits non liquidés avec la famille d'origine (Boszormenyi-Nagy, 1965; Friedman, 1980). Toutefois, Bloom (1977) affirme que la perception des parents concernant l'expérience de séparation d'avec leur famille d'origine combine la réalité perçue et remémorée de l'enfance et sa réévaluation en fonction des événements expérimentés depuis. Lowenthal et al. (1975) ainsi que Peskin (1977) notent aussi l'influence des besoins et circonstances présentes.

\section{Cas 4: La famille D}

La famille $D$ se compose de six membres dont quatre enfants: Sylvie ( 26 ans), Louis ( 25 ans), Jean ( 23 ans) et René ( 21 ans). Le départ de Madame de la maison parentale, à 20 ans, s'insère dans une série d'événements familiaux importants. Depuis l'âge de 12 ans, à cause de la santé précaire de sa 
mère, Madame assumait la direction de la maison et faisait la cuisine pour les $25 \mathrm{em}$ ployés de son père. Lorsqu'elle atteint l'âge de 18 ans, sa mère meurt, la laissant chef incontesté. La séparation fut précipitée par les mariages consécutifs de son père et de son frère et donc par l'arrivée de deux autres femmes. Madame, ne pouvant tolérer d'être reléguée au second plan, choisit de s'installer à environ 200 milles de la maison. Elle se marie un an plus tard. Pour sa part, Monsieur se sépare de sa famille à l'âge de 14 ans, un mois après la mort de sa mère.

A 21 ans, Sylvie quitte le domicile familial pour aller cohabiter avec son ami. Cette séparation correspond à une période particulière dans la vie de Madame, au moment où celle-ci était au chevet de son père gravement atteint d'un cancer terminal des poumons. Depuis déjà trois mois, Madame demeurait dans la maison paternelle. Le départ de Sylvie avait toutefois été discuté avant que Madame ne quitte. Bien qu'elle soit la première à partir du domicile, Madame ne s'en est pas trop rendue compte devant l'impact du décès de son père. Ce départ coincide aussi avec son retour sur le marché du travail.

Jean se marie à 19 ans, provoquant choc et surprise. L'annonce de la cérémonie s'effectue deux mois seulement avant l'événement. Madame exprime certaines réticences face à sa belle-fille.

A 20 ans, Louis quitte dans un climat amer et chaotique. Bien qu'ayant toujours eu d'excellentes relations avec sa famille, les circonstances entourant son départ créent des tensions familiales qui persisteront pendant plusieurs mois. Son départ fait suite (1 mois) à une escapade d'une fin de semaine avec l'amie de son frère cadet. Une importante distance est engendrée entre sa famille et lui, ce qui toutefois n'empêchera pas sa cohabitation avec son amic. La nature de ses relations avec sa famille s'exprime par la proximité ou la distance physiques qu'il place entre eux. Au moment où leurs rapports sont les plus tendus, il habite dans une ville voisine et ne côtoie ses parents que très rarement. Lorsque leurs relations s $s^{\prime} \mathrm{a}$ méliorent, il déménage avec son amie à l'ê. tage supérieur de l'appartement où résident ses parents.

René, à 20 ans, se sépare d'avec sa famille d'origine pour aller cohabiter avec son amie. Ce départ initial se caractérise par des départs-retours que René effectue entre ses parents et son amie. René étant le dernier à quitter la maison, il semble éprouver des difficultés à laisser ses parents seuls. Pour leur part, Madame et Monsieur affirment préférer la cohabitation qui leur apparait comme réversible: ils présentent certains problèmes à accepter les départs comme définitifs. Nous remarquons done combien l'attitude de René dépend de difficultés inavouées et sous-jacentes quant à la séparation dans le système familial.

\section{DISCUSSION}

L'examen des différents cas permet de voir l'importance du rite de passage dans la séparation parents-enfants. Bien que la plupart des enfants partent avant, le mariage confère aux yeux des parents un statut nouveau, l'accès au monde adulte. Pour les parents, le fait de jouer un rôle complet dans la société s'avère ainsi intimement lié au mariage, qui apparait comme la transition déterminante. Le travail régulier et permanent peut jusqu'à un certain point remplir également cette fonction particulière: il constitue un rite de passage socialement admis comme gage de maturité.

Mais quelles sont les raisons assurant au mariage ce rôle de rite de cycle de vie par excellence? Dans notre société, nous pouvons supposer que l'une des fonctions possibles de la cérémonie du mariage soit de faciliter une modification d'attitudes envers la sexualité (Kitahara, 1974). De fait, lors de l'entrée dans la vie de couple, une interdiction formelle des rapports sexuels préconjugaux devient brusquement une permission et même une exigence. La cérémonie du mariage prépare donc les acteurs à des relations hétérosexuelles et proclame officielle- 
ment le droit à une génitalité adulte, tout comme les rites de l'adolescence dans certaines tribus, tels que décrits par Vizedom (1976). Il s'y opère une séparation d'avec le monde asexuel suivie d'une incorporation dans le monde de la sexualité (Van Gennep. 1909).

Comparativement, l'accès officieux à des relations hétérosexuelles avec la cohabitation comporte des aspects difficiles à acalors au niveau de la transition. Les rites d'incorporation n'ont pas été amorcés. Par opposition, l'efficacité du rite de transition que représente le mariage est appuyée par la loi et la coutume. Les participants sont considérés comme changés simplement en vertu de leur rôle dans le rituel. Ce dernier leur confere des droits et exigences irréversibles.

De ce qui précède se dégage l'importance d'une démarcation claire et précise concernant les modalités du départ de la maison. Les parents expriment leur satisfaction lorsqu'ils se retrouvent devant des rites établissant sans l'ombre d'un doute la croissance ou la maturité. Le mariage, l'obtention d'un dimplôme ou d'un poste rémunéré à temps plein sont des exemples de tels événements. Nous relevons en effet que, lorsque les départs sont réalisés dans un contexte autre que le mariage, les parents mentionnent plus fréquemment leur participation active lors des diverses tâches entrainées par le déménagement. Cette implication vise vraisemblablement la clarification et la mise en branle d'un rite de passage mieux délimité, facilitant ainsi le processus de séparation.

Le rite de passage retenu ne parait pas indépendant de l'âge de l'enfant. L'échantillon étudié démontre une différence d'un peu plus de deux ans entre la moyenne d'âge des enfants quittant pour le mariage (21.65) et celle des enfants partant pour des motifs autres (19,5), Nous pouvons nous demander si le choix d'un mode moins définitif de séparation n'est pas lié à l'âge (la maturité) auquel il est entrepris. En ce sens, la cohabitation permettrait une séparation progressive, peut-être plus adaptée au contexte social actuel (Bloom, 1977). En effet, si nous observons les personnes optant pour ce mode de vie, nous remarquons qu'elles l'emploient souvent comme un étape transitionnelle menant éventuellement à un état plus certain. L'union libre paraitrait done une solution efficace au départ de la maison puisqu'elle permettrait une séparation d'avec la famille d'origine sans toutefois exiger un changement définitif et irréversible.

\section{Conclusion}

Du point de vue des parents, le mariage demeure le rite depassage par excellence lors du départ des enfants de la maison, afin de délimiter la réelle séparation parentsenfant. Nous ne pouvons toutefois que noter la popularité grandissante de l'union libre. Cette nouvelle tendance peut découler de difficultés engendrées par notre contexte social, principalement en ce qui a trait au processus de séparation parents-enfant. La pénurie de rituels et de lignes de conduite pour les rites du cycle de vie familiale ainsi que le délai et la rapidité du processus de séparation (comparativement aux cultures antérieures) peuvent être responsables de ce phénomène. Nous pouvons supposer que la cohabitation sera favorisée dans les familles exprimant des réticences quant à la séparation. Cette option favoriserait effectivement un détachement progressif, plus sécurisant et réversible entre les enfants et leur famille d'origine. Cette situation s'avère toutefois 
incomplète puisque l'individu demeure en état de transition, privé de statut clairement défini. Dans une situation "non pathologique"., l'enfant abandonnera cette fixation à une période transitionnelle et optera éventuellement pour un rite complet de passage. qui lui permettra de finaliser la séquence en trois mouvements de Van Gennep (1909).

L'étude des rites du cycle de vie à la période postparentale fait ressortir le peu d' information disponible actuellement, malgré l'importance qu'ils revêtent dans l'évolution familiale, tel que le laisse supposer cette étude exploratoire. Des travaux ultér- ieurs semblent donc nécessaires, examinant à la fois les perceptions des parents et des enfants. Une connaissance adéquate de ces processus ne pourrait qu'améliorer la com. préhension des dynamiques de la vie fami. liale, permettant ainsi une meilleure intervention. En ce sens, les moments précédant et suivant les rites du cycle de vie peuvent se révéler des périodes privilégiées de thérapie. Ils donnent la possibilité de retravailler les triangles, l'objectif étant de développer des soi plus différenciés dans les relations parents-enfant permettant une séparation réelle et adaptative.

\section{ABSTRACT}

This exploratory clinical study examines the circumstances surrounding the departure of a family member at the time of marriage. An in-depth analysis of 10 case studies of parents suggests that marriage is an excellent rite of passage for the delineation of parentchild separation. Cohabitation fosters a progressive detachment but remains a period of transition. The choice of the rituals for the marriage ceremony provides an index of the fluctuations occurring in the family system.

\section{REFERENCES}

Bloom, M. V. The Death of Childhood: The Process of Adolescent Parental Separation, Dissertation submitted in partial fulfilment of the requirements for the degree of doctor of philosophy, Berkeley, Wright Institute, 1977.

Boszormenyi-Nagy, I. "A Theory of Relationships: Experience and Transaction" in 1. Boszermenyi-Nagy and J.L. Framo (eds.), Imtensive Family Therapy. New York, Harper and Row, 1965.

Friedman, E.H. "'Systems and Ceremonies: Family View of Rites of Passage "In E.A. Carter and M. McGoldrick (eds.), The Family Life Cycle: a Framework for Fantily Therapy, New York. Gardner Press, 1980.

Hunchett, S, and Casale, L., "The Theory of Transi. tional Phenomena and Cultural Symbols" Contemporary Psychoanalysis. 12:496-507, 1976.

Kitahara, M. "A. Function of Marriage Ceremony" Anthropologica, 16: 163-175, 1974.

Lefebvre, G.. Adaptution du couple à la période postparentale. Mémoire inédit de maitrise en psy. chologie, Université de Montrèal, 1982.

Levy-Strauss, C. Structural Anthropology, New York. Busic Books, 1963.
Linton, R., "Age and Sex Categories" Am. Soc. Rew $7: 589-603,1942$.

Lowenthal, M.F. Chiriboga, D and Thurnher, M. Four Stages of Life: a Camparative Study of Women and Men Facing Transitions, San Francisco, Jossey-Bass, 1975.

Peskin, H., "Personal Communications" In M.V. Bloom. The Death of Childhood, Berkeley, Wright Institute, 1977.

Stierlin. H. Separating Parents and Adolescents: A Perspective on Rumning away. Schizophrentia. and Waywandness, New York, Qundrangle. 1974.

Van Gennep, A, Les Riter de Passage, Paris, Ferme. 1909.

Van Gennep, A. The Rites of Passage, Chicago, The Untiversity of Chicago Press, 1960.

Vizedom, M. "Rites and Relationships: Rite of Passage and Contemporary Anthropology" Sage Research Papers in the Social Sciences 4 (90. $027)=1-63,1976$.

Yeats, E. L. Family Rites of Passage: A Study of Ritual and the School Entry Transition in Fwe Healfiry Families. Dissertation submitted in partial fulfilment of the requirements for the degree of Doctor in Philosophy, Massaschusetts. Univer. sity of Massischusetts. 1979. 